\title{
LA EDUCACIÓN SUPERIOR EN MÉXICO TENDENCIAS Y DESAFÍOS
}

\author{
YAZMín CRuz LóPEZ*1 \\ Anna Karina Cruz LóPez ${ }^{* * 2}$
}

\begin{abstract}
* Ingeniera Civil del Instituto Tecnológico de Estudios Superiores de Monterrey (ITESM), tiene un MSc. en Ingeniería Ambiental con especialidad en prevención y control de la contaminación también del ITESM. Master en Gestión de Residuos Industriales y Municipales en la Fundación UPC. Su investigación doctoral se centra en Universidades y Desarrollo Sostenible. Ha sido coordinadora del Programa de Ecoeficiencia del Consejo para el Desarrollo Sostenible de América Latina (CEDSAL). Consultora y Auditora de ISO 14001 para ABS Group Services de México. En la UPC ha colaborado desde el 2003 hasta el 2005 en el Centro Interdisciplinario de Tecnología, Innovación y Educación para la Sostenibilidad. Desde el año 2005 es Coordinadora del informe La Educación Superior en el Mundo 2007 y miembro del Secretariado de la GUNI. Email: Yazmin.Cruz@upc.edu

** Ingeniera Mecánica Electricista del Instituto Tecnológico de Estudios Superiores de Monterrey (ITESM), tiene una Maestría en Administración de Tecnologías de Información también del ITESM. Ha realizado además estudios de doctorado en Ingeniería Industrial con especialidad en tecnologías de información. Se ha desempeñado como Coordinadora de la Universidad Virtual del ITESM en el Campus Colima. Ha sido Coordinadora de Ingeniería Industrial de la Universidad del Mayab, además de Directora de Servicios Académicos de la misma institución. Asimismo, ha impartido las asignaturas de tecnologías de información, ingeniería de materiales y diseño de herramientas a nivel licenciatura y de selección uso y diseño de recursos didácticos, tecnología y educación, educación efectiva a distancia a nivel postgrado. Actualmente es consultora de modelos y procesos de la división de universidades del grupo Integer, que promueve y asesora proyectos educacionales que abarcan todas las etapas educativas y formativas, desde el preescolar hasta la especialización profesional en más de15 países alrededor del mundo.
\end{abstract}

Resumen: Este capítulo hace un análisis del sistema mexicano de Educación Superior en la última década y de los principales desafíos a que se enfrenta.

Palabras claves: Educación Superior. México. Cifras del sistema de ES mexicano. Políticas de equidad. Garantía de calidad. Retos del sistema.

Abstract: This chapter analyzes the Mexican system of higher education in the last decade and discusses the major challenges this system faces.

Keywords: Higher education. Mexico. Numbers of the Mexican system of higher education. Equity policies. Quality assurance. Challenges.

\section{Introducción}

La forma de organización política de México es la de una República representativa, democrática, federal, compuesta de 31 Estados libres y soberanos en todo lo concerniente a su régimen interior; pero unidos en una federación. El Distrito Federal (DF), es sede de los Poderes de la Unión y Capital de los Estados Unidos Mexicanos.

México cuenta con una población de más 103 millones de habitantes, de acuerdo con el Instituto Nacional de Estadística, Geografía e Informática

1-2 Las autoras son responsable de la selección y la presentación de los hechos, las declaraciones y conclusiones incluidas en su artículo. Estas no reflejan necesariamente las opiniones de la GUNI y/o del Grupo Integer. 
(2005). Del total, más de 53 millones son mujeres y 50.3 millones son hombres. Además, por su origen histórico, más de 10 millones del total de la población pertenecen a grupos indígenas.

En las últimas dos décadas el país ha experimentado un importante proceso de reestructuración, el cual ha modificado las tendencias tanto de la distribución geográfica de las actividades económicas como de la población, pasando de un patrón altamente concentrado en unas cuantas ciudades y regiones ( $76 \%$ de la población vivía en localidades urbanas en 2005), a uno un poco más diversificado, sin embargo esto no ha repercutido en el equilibrio de beneficios pues las principales actividades económicas siguen desarrollándose en los centros urbanos y se refieren a la industria terciaria (comercios y servicio).

La apertura económica hacia el exterior, característica principal del nuevo modelo de desarrollo adoptado por el país, ha tenido como resultado el desplazamiento de algunas ramas industriales importantes del centro hacia el occidente y norte del país, el surgimiento y consolidación de polos turísticos en las costas y el dinamismo de la maquila de exportación en las ciudades de la frontera norte.

Aunque hay una desaceleración del crecimiento de las principales urbes del país y la reorientación de los flujos migratorios hacia las ciudades medias y pequeñas, el patrón de distribución territorial de la población continúa siendo polarizado. En 2000, la tercera parte de la población nacional se concentraba en nueve ciudades mayores de un millón de habitantes, mientras que en el otro extremo, la cuarta parte de los mexicanos habitaba en 196 mil localidades menores de 2.500 habitantes. Es decir, se mantiene una alta concentración de población en un número reducido de ciudades, y una gran dispersión de la población en miles de localidades pequeñas.

Uno de los principales retos sociales es el que se refiere a la distribución de la riqueza, en México el decil más rico de la población percibe el $43.1 \%$ de los ingresos, mientras que el $20 \%$ mas pobre percibe el $3.1 \%$ de los ingresos. La mitad de la población mexicana vive en pobreza y un quinto en pobreza extrema (Banco Mundial 2002).

Como el resto de los países latinoamericanos el crecimiento económico de México (representado por el crecimiento promedio del PIB) se encuentra por debajo de los países asiáticos y dentro del bloque de países latinoamericanos por debajo de países como Chile, Costa Rica, Colombia y Brasil. Al PIB nacional el DF aporta el 22.8\% mientras que estados como Tlaxcala, Nayarit y Colima aportan tan solo el $0.5 \%$ (INEGI 2004).

La inequidad a lo largo del territorio mexicano tiene muchas caras, por 
ejemplo el analfabetismo. Aunque la media nacional (INEGI 2005) indica que solo el $8.4 \%$ de la población mayor de 15 años es analfabeta, existen estados como Chiapas, Oaxaca y Guerrero en donde el porcentaje aumenta al 20-21\% de la población.

En estos mismos estados solamente el 29-38\% de la población tiene acceso a agua potable y la esperanza de vida es un año menor que la media nacional.

Adicionalmente, en la última década desastres naturales graves como los huracanes Isidore, Wilma y las inundaciones del 2007 en los estados de Tabasco y Chiapas han sometido varias regiones a situaciones de devastación, crisis y reconstrucción.

\section{Educación Superior en México}

México tiene una larga tradición e historia en ES, la universidad fue una de las primeras instituciones que se creo después de la conquista. Fundada en 1551, la Real y Pontificia Universidad de México, se convertiría en la Universidad Nacional Autónoma de México (UNAM). Como en la mayoría de los países de la región en la segunda mitad del Siglo XX se experimentó un crecimiento sin precedentes en el ámbito de la ES, tanto en el tipo de instituciones, como en el número de estudiantes, profesores y áreas de investigación. En la actualidad, la ES se concibe como un instrumento vital para la modernización de México.

El Programa Nacional de Educación 2001-2006 ofrece una visión de la educación superior al año 2025:

- La educación superior será la palanca impulsora del desarrollo social, de la democracia y la convivencia multicultural. Proporcionará a los mexicanos los elementos para su desarrollo integral y formará científicos, humanistas y profesionales, en todas las áreas del saber, portadores de conocimientos de vanguardia y comprometidos con las necesidades del país.

- Para el primer cuarto del siglo, el sistema de educación superior estará conformado por 32 sistemas estatales, contará con un amplio respaldo por parte de la sociedad y atenderá a más de la mitad de la población entre 19 y 23 años con una oferta amplia, flexible y diversificada de programas educativos en instituciones de diversos perfiles tipológicos. Además, ofrecerá oportunidades de actualización a todos sus egresados y contará con una oferta variada y modalidades adecuadas de educación continua para satisfacer necesidades educativas de los adultos. Una de las características del sistema será su coordinación con los otros tipos 
educativos y con los ámbitos de la ciencia, la tecnología, el arte y la cultura, así como la operación de amplias redes de cooperación e intercambio académico en el nivel nacional e internacional, que sustentarán los programas de movilidad de profesores y alumnos.

- La sociedad estará plenamente informada del desempeño académico y del uso de los recursos de todas las instituciones de educación superior, con sustento en procesos consolidados de evaluación y acreditación.

Para construir esta visión se necesita el compromiso de las instituciones involucradas, la participación entusiasta de las comunidades educativas y el apoyo de las autoridades federal y estatales. No es una tarea fácil e implica grandes y complejos esfuerzos.

\subsection{Características del sistema de ES en México}

El sistema de ES en México se caracteriza por su gran magnitud y diversidad, además es complejo y heterogéneo debido al tamaño y las particularidades que lo integran, así como por las características del profesorado. El marco normativo básico de la educación superior en México lo conforman:

1. Constitución Política de los Estados Unidos Mexicanos,

2. Ley General de Educación,

3. Ley para la Coordinación de la Educación Superior,

4. Reglamentaria del Artículo 5to. Constitucional,

5. Leyes estatales de educación superior,

6. Reglamento Interior de la Secretaría de Educación Pública (SEP),

7. Leyes orgánicas de las universidades públicas autónomas y no autónomas,

8. Decretos gubernamentales de las universidades no autónomas,

9. Acuerdos 93, 243, 279, 286 y 328 de la SEP,

10. Convenios de coordinación, operación y apoyo financiero entre la $\mathrm{Fe}$ deración, estados e instituciones,

11.Ley Federal del Trabajo que rige las relaciones de trabajo en las IES.

El Poder Ejecutivo es responsable de elaborar el Plan Nacional de Desarrollo con una vigencia sexenal. El programa sectorial asociado a la educación lo elabora la SEP, este programa es de observancia obligatoria y contiene los objetivos estratégicos, políticas, objetivos particulares, líneas de acción y metas para el periodo correspondiente. El Gobierno Federal establece los planes y las políticas nacionales y los gobiernos estatales los planes y políticas en el ámbito de sus competencias. 


\subsection{Tipología de las instituciones}

Las IES pueden clasificarse también en términos de la naturaleza de su oferta educativa y de las funciones que en ellas se realizan. La Asociación Nacional de Universidades e Instituciones de Educación Superior (ANUIES) desarrolló en 1999 una clasificación de las IES que ha sido de gran utilidad. La descripción de las tipologías que aquí se presentan está basada en la propuesta de la ANUIES y la que utiliza la SEP.

1. Subsistema de universidades públicas federales: Son el conjunto de IES con fondos federales o estatales pero gobernadas por si mismas, la mayoría de ellas son autónomas. Las instituciones que conforman este subsistema realizan, además de las funciones de docencia, un amplio espectro de programas y proyectos de investigación (generación y aplicación innovadora del conocimiento), y de extensión y difusión de la cultura.

2. Subsistema de universidades públicas estatales: Son el conjunto de IES con fondos estatales pero gobernadas por si mismas, la mayoría de ellas son autónomas. Estas instituciones son organismos descentralizados de los gobiernos de los estados y desarrollan las funciones de docencia, generación y aplicación innovadora del conocimiento, así como de extensión y difusión de la cultura.

3. Subsistema de educación tecnológica: La mayoría de ellas son coordinadas por el Gobierno Federal a través de la Subsecretaría de Educación e Investigación Tecnológicas de la SEP, las restantes son institutos descentralizados de los gobiernos estatales.

4. Subsistema de otras instituciones públicas: Incluye a las instituciones dependientes de la SEP y de otras secretarías de estado.

5. Universidades tecnológicas públicas: Son organismos públicos descentralizados de los gobiernos estatales y en ellas se realizan las funciones de docencia, aplicación del conocimiento, extensión y prestación de servicios tecnológicos. Ofrecen programas educativos en regiones donde no existía oferta educativa del tipo superior, y operan bajo la responsabilidad de la universidad tecnológica con autorización de su Consejo Directivo. Se imparten exclusivamente programas de dos años de duración, que conducen a la obtención del título de técnico superior universitario (dicho tipo de programas tan bien puede ser ofertados por otros tipos de IES). 
6. Universidades politécnicas públicas: Son organismos descentralizados de los gobiernos de los estados. Este perfil de institución se incorporo al sistema de ES, en el 2002, con el propósito de ampliar las oportunidades de acceso a la educación superior pública y fortalecer la pertinencia de la oferta educativa de las regiones en las que han sido ubicadas.

7. Subsistema de universidades públicas interculturales: Creado por iniciativa del Gobierno Federal en el periodo sexenal 2000-2006, son organismos descentralizados de los gobiernos de los estados y están localizadas en regiones con alta densidad de población indígena pero abiertas a todo tipo de estudiante. Ofrecen opciones educativas innovadoras, bajo un enfoque intercultural, para atender necesidades y potenciar el desarrollo de las regiones en que están ubicadas. Las actividades de generación del conocimiento se desarrollan en los campos de lengua y cultura indígenas, y desarrollo regional sustentable.

8. Instituciones particulares: IES con financiación privada, autónomas en su gestión y constituidas con personalidad jurídica. Los estudios impartidos requieren, del Reconocimiento de Validez Oficial de Estudios (RVOE) de la SEP o de los gobiernos de los estados o, bien, estar incorporados a una institución educativa pública facultada para ello.

9. Instituciones de formación docente: IES públicas y particulares responsables de formar a profesionales para actividad docente en los distintos tipos y niveles del Sistema Educativo Nacional en áreas como educación preescolar, en educación primaria, en educación secundaria, en educación especial y en educación física.

10. Subsistema de centros públicos de investigación: Se integra por instituciones que ofrecen programas académicos básicamente de posgrado y, en menor medida, programas de licenciatura. La coordinación de estos centros está bajo la responsabilidad del Consejo Nacional de Ciencia y Tecnología (CONACYT), el cual establece las políticas para su desarrollo y asigna los recursos para su operación.

11. Otras instituciones públicas: Existen otras instituciones de educación superior públicas autónomas y no autónomas, no incluidas en los subsistemas anteriores, sectorizadas en diversas secretarías federales o que son organismos descentralizados o desconcentrados de los gobiernos de los estados (universidades, colegios, centros de investigación y 
estudios avanzados, escuelas de música, centros de educación en artes, etc.).

\subsection{Dimensión del sistema de ES}

En los últimos años el sistema de ES se ha modificado sustancialmente, su tamaño, su composición y los modelos educativos utilizados. Por ejemplo, a partir de 1999 existen un mayor número de IES particulares que públicas, atendido en su mayoría por personal académico por horas. Presta servicios a una población estudiantil con una distribución cada vez más equilibrada según sexo, $51.5 \%$ hombres vs. $48.5 \%$ mujeres, y con una oferta educativa concentrada en centros urbanos. La Tabla 1 ilustra el número de instituciones y estudiantes por subsistema en el 2005.

Tabla 1. Número de instituciones y estudiantes por subsistemas, 2005

\begin{tabular}{l|r|r|r|c}
\hline Subsistema & $\begin{array}{c}|c| \\
N^{\circ} \text { de } \\
\text { instituciones }\end{array}$ & \multicolumn{1}{c|}{$\%$} & Matrícula & $\%$ \\
\hline Universidades públicas federales & 4 & 0,2 & 307.778 & 12,1 \\
\hline Universidades públicas estatales & 46 & 2,4 & 785.917 & 31,0 \\
\hline Institutos públicos tecnológicos & 211 & 11,2 & 325.081 & 12,8 \\
\hline $\begin{array}{l}\text { Universidades públicas } \\
\text { tecnológicas }\end{array}$ & 60 & 3,2 & 62.726 & 2,5 \\
\hline $\begin{array}{l}\text { Universidades públicas } \\
\text { politécnicas }\end{array}$ & 18 & 1,0 & 5.190 & 0,2 \\
\hline $\begin{array}{l}\text { Universidades públicas } \\
\text { interculturales }\end{array}$ & 4 & 0,2 & 1.281 & 0,05 \\
\hline $\begin{array}{l}\text { Instituciones públicas de formación } \\
\text { docente }\end{array}$ & 249 & 13,2 & 92.041 & 3,6 \\
\hline $\begin{array}{l}\text { Instituciones privadas (universidades, } \\
\text { institutos, centros y academias) }\end{array}$ & 995 & 52,6 & 776.555 & 30,6 \\
\hline $\begin{array}{l}\text { Instituciones privadas de formación } \\
\text { docente }\end{array}$ & 184 & 9,7 & 54.267 & 2,1 \\
\hline Centros públicos de investigación & 27 & 1,4 & 2.801 & 0,11 \\
\hline Otras instituciones públicas & 94 & 5 & 124.609 & 4,9 \\
\hline \begin{tabular}{l} 
Total \\
\hline
\end{tabular} & 1.892 & 100 & 2.538 .256 & 100 \\
\hline
\end{tabular}

Fuente: Sobre la base de Secretaría de Educación Pública, México - Country Background Report, 2006, pp.43-50. Disponible en: http://www.oecd.org/dataoecd/22/45/37746065.pdf 
De la matrícula de educación superior, $39.9 \%$ corresponde al sostenimiento autónomo; $12.6 \%$, al estatal; el sostenimiento federal cubre $14.8 \%$, y las instituciones particulares cuentan con 32.7 por ciento.

La educación superior se distribuye de la siguiente manera:

a) Profesional asociado o Técnico Superior: $3.3 \%$,

b) Licenciatura: $90.4 \%$

c) Posgrado: $6.3 \%$

Dentro de la ES se ubica la educación normal, que cubre el 5.7\% de la matrícula total de este tipo educativo; se imparte en sus opciones de normal licenciatura en educación preescolar, educación primaria, educación secundaria, educación especial y educación física.

Existen diferencias bastante notorias en la oferta por entidad federativa, sobre todo por nivel de estudios, de tal forma que aunque el DF solo concentra $13.4 \%$ de la oferta de licenciatura, centraliza $21.7 \%$ de maestría y $50.4 \%$ de la oferta de programas doctorales.

Por área de estudio, los programas de licenciatura que atienden la mayor cantidad de población estudiantil son aquellos referentes a las ciencias sociales y administrativas, mientras que el área de ciencias naturales y exactas es la que atiende al menor número de alumnos y genera el menor número de titulados. Dicho comportamiento se repite en los programas de maestría, mientras que en los programas doctorales la mayoría de los egresados provienen del área de ingeniería y tecnología.

La oferta de programas con modalidades educativas emergentes como la educación semiescolarizada, abierta y a distancia se encuentra en franco crecimiento, sobre todo para el nivel de postgrados, como respuesta a un mercado demandante con alta capacidad adquisitiva pero poca disponibilidad de tiempo y de movilidad. Mientras que los programas de licenciatura ofertados en estas modalidades, responden a la necesidad de certificar competencias profesionales de adultos que ya se encuentran activos en el campo laboral o que habitan en comunidades con una escasa o nula oferta de ES.

El personal docente que participa en la ES había mantenido una tendencia positiva en su variación porcentual anual, excepto para el período 2000/2001, donde se presentó una disminución de 4.6. Durante el ciclo escolar 2005-2006, la plantilla docente estaba formada por 259,884 profesores de los cuales $39 \%$ labora en las ES particulares. En los últimos años se han implementado varios programas que buscan capacitar al profesorado, entre ellos el Programa 
Integral para el Fortalecimiento del Posgrado (PIFOP), uno de los proyectos de excelencia académica del CONACYT y cuyo propósito es apoyar los estudios de posgrado de las universidades públicas.

Asimismo, durante la última década han nacido numerosas asociaciones, consorcios y redes con el objetivo de desarrollar sinergias entre las IES en diferentes temas. Por ejemplo, agrupaciones no gubernamentales como la ANUIES o la Federación de Instituciones Mexicanas Particulares de Educación Superior (FIMPES) que agrupa a las IES particulares y certifica su calidad institucional. Consorcios educativos y redes universitarias que agrupan a un conjunto IES que comparten un mismo modelo educativo y operativo, ya sea en una sola personalidad jurídica como el sistema del Instituto Tecnológico de Estudios Superiores de Monterrey (ITESM) o la Universidad del Valle de México (UVM), o conservando su independencia, como la Red de Universidades Anáhuac.

\subsection{Políticas de equidad, acceso y admisión}

Vista en perspectiva histórica, la ES ha creado oportunidades de desarrollo personal, movilidad social y crecimiento económico para varias generaciones en México. Esto ha contribuido de forma importante al desarrollo del país. Sin embargo las oportunidades educativas continúan siendo escasas en relación con la demanda y mal distribuidas en el territorio nacional, pues aún no se encuentra disponible sobre todo para los grupos más marginados y en especial en el área rural.

La equidad es una prioridad de la política de la ES en México. Las decisiones sobre la ampliación del sistema ES buscan favorecerla, en especial la participación de los estudiantes de grupos indígenas, marginados económicamente y mujeres. Estas medidas se apoyan en dos esquemas, el programa de becas en función de los ingresos (PRONABES) y la creación de nuevas instituciones en regiones marginadas, como universidades tecnológicas, intercultutrales, institutos tecnológicos y universidades politécnicas. Sin embargo sigue siendo necesario fomentar el crecimiento de la cobertura privilegiando la equidad sin poner en riesgo los avances en materia de calidad.

Durante la década de los 90's 12 de cada 100 personas entre los 19 y los 23 años de edad tenía acceso a la ES, actualmente se calcula de uno de cada cinco personas en el rango de edad puede acceder a la ES, por ello se habla de un sistema masificado. La población estudiantil de las IES ha mantenido un ritmo de crecimiento exponencial positivo, excepto en lo referente a la educación normal en donde a partir del ciclo escolar 2000/2001 el total de 
alumnos ha disminuido anualmente. En el ciclo escolar 2005-206 ingresó el $79.9 \%$ de los egresados del ciclo anterior de la educación media superior. La matrícula escolarizada equivale a $23.7 \%$ de la población de 19 a 23 años de edad excluyendo al posgrado. Sin embargo es un porcentaje engañoso, las oportunidades educativas de un joven del DF son cuatro veces más elevadas que las de un joven que vive en Chiapas (Schmelkes, 2005).

Debido a los problemas de financiación de las IES públicas se han establecido mecanismos restrictivos de admisión. Actualmente se utilizan exámenes estandarizados, según el resultado se ubica a los aspirantes hasta llenar el cupo establecido por la institución. Se han iniciado "semestres cero", cuyo objetivo es ofrecer procesos de regularización o afianzamiento de conocimientos y habilidades previas al inicio formal de los estudios. Además hay plazos de permanencia de los estudiantes en la IES.

En el subsector de instituciones privadas, se emplean también exámenes estandarizados. Suele haber preferencia por los estudiantes que cursaron la educación media en las propias instituciones que cuentan con este servicio, exigiendo ciertas condiciones académicas. Hay una parte del subsector de instituciones privadas que tiene escasos o nulos procesos de selección.

\subsection{Financiación}

En términos generales las instituciones públicas reciben del Gobierno Federal un subsidio anual ordinario para el desarrollo de sus funciones. El subsidio de las universidades públicas estatales se integra con aportaciones diversas, resultado de las negociaciones entre el Gobierno Central y de los gobiernos del estado en el que se encuentra ubicada la institución. El subsidio de los institutos tecnológicos federales se asigna por la SEP con base en un conjunto de lineamientos y criterios previamente establecidos. En el caso de las universidades tecnológicas, politécnicas, interculturales e institutos tecnológicos estatales, el subsidio que reciben es 50\% federal y 50\% estatal. El mismo esquema se aplica a las universidades públicas no autónomas estatales creadas a partir de 1977.

México dedica $6.3 \%$ del PIB como gasto público en instituciones y en administración educativas, a la ES le corresponde 1.4\% gasto público como $\%$ del PIB (UNESCO, 2006). En 2005, de acuerdo con cifras de la SEP, el monto total para la ES fue de 29'395,671.1 pesos mexicanos, de los cuales la Federación aportó $65.5 \%$ y los gobiernos estatales $34.5 \%$.

En el caso de las instituciones privadas, su principal fuente de ingresos deriva de las cuotas que cobran a los estudiantes. Las donaciones filantrópicas 
existen, pero no son de la magnitud e importancia que tienen en otros países. Si bien el subsector de las instituciones privadas recibe también fondos de origen público, éstos no constituyen una fuente relevante en el ingreso total de estas instituciones (CINDA, 2007).

Además se han establecido programas especiales, cuyos fondos se incluyen en la categoría de subsidio extraordinario destinados a mejorar y asegurar la calidad de la educación superior mediante los siguientes programas:

- Fondo para la Modernización de la Educación Superior (FOMES): orientado a contribuir con los objetivos de los Programas Integrales para el Fortalecimiento de las Universidades Públicas. Los fondos del FOMES se distribuyen entre universidades públicas estatales, universidades públicas estatales de apoyo solidario, y universidades públicas federales.

- Programa de Mejoramiento del Profesorado (PROMEP): el objetivo es alcanzar estándares internacionales tanto en la formación como en el desempeño del personal académico de carrera. Estos fondos se orientan a universidades públicas estatales, universidades públicas estatales de apoyo solidario y universidades públicas federales.

- Programa de Apoyo al Desarrollo Universitario (PROADU): diseñado para apoyar acciones puntuales de colaboración nacional e internacional en los cuerpos académicos. Distribuye apoyos puntuales a universidades públicas estatales, universidades públicas estatales de apoyo solidario, universidades públicas federales, universidades tecnológicas, otras instituciones de educación superior y asociaciones científicas y tecnológicas.

- Fondo de Inversión para las Universidades Públicas Estatales de programas evaluados y acreditados (FIUPEA): tiene la finalidad de coadyuvar con los objetivos de los Programas Integrales de Fortalecimiento de las Universidades Públicas, fomentando el aseguramiento de la calidad de los programas educativos que han sido reconocidos por parte de los organismos competentes para ello.

- Programa Integral de Fortalecimiento del Postgrado (PIFOP): está orientado a la acreditación de los programas de estudio de posgrado y se realiza a través de comités de pares que certifican las condiciones 
necesarias para ser considerados de calidad, o bien de calidad internacional.

- Programa Integral de Fortalecimiento Institucional (PIFI): distribuye a las universidades públicas fondos del Gobierno Federal desde el año 2001, con el objetivo de mejorar la calidad de sus programas educativos y de los servicios que ofrecen, así como la calidad de los programas acreditados por organismos especializados, o de los procesos de gestión que han sido certificados por las normas correspondientes (Normas ISO-9000). La operación del PIFI persigue dos objetivos generales: 1) la mejora del sistema público de educación superior mediante el fortalecimiento y desarrollo de las IES que lo integran; y 2) el logro del reconocimiento social de las IES como resultado de la mejora de sus indicadores de desempeño y la transparencia de su operación. Es de destacar que los miembros de la ANUIES son candidatos a recibir fondos PIFI.

- Fondo de Apoyo Extraordinario a las Universidades Públicas (FAEUP): creado en el año 2002 con el propósito de apoyar la realización de proyectos formulados por las universidades públicas estatales e instituciones afines que permitan incidir en la solución, a mediano y largo plazos, de problemas estructurales de carácter financiero.

Los cambios más importantes en las modalidades de financiamiento a las universidades públicas han consistido en la emergencia de toda una serie de fondos especiales, sometidos a concurso, con los cuales dichas instituciones amplían su margen de maniobra para desarrollar ciertos proyectos.

\subsection{Sistema de garantía de la calidad}

Las primeras acciones para la evaluación de la educación superior en México se realizaron en la década de los setenta y fueron parte de programas de gobierno e iniciativas de la ANUIES.

La evaluación de la educación superior se institucionalizó con el Programa para la modernización educativa 1989-1994 del Gobierno Federal. En este programa, se establecieron como acciones prioritarias las evaluaciones interna y externa permanentes de las instituciones para impulsar la mejora de la calidad de los programas educativos y de los servicios y como meta la creación de una 
instancia que integrara y articulara un proceso nacional de evaluación de la educación superior.

Para lograr este objetivo, la Coordinación Nacional para la Planeación de la Educación Superior (CONPES) creó en 1989 la Comisión Nacional de Evaluación de la Educación Superior (CONAEVA), la cual diseñó la estrategia nacional para la creación y operación del Sistema nacional de evaluación de la educación superior, sustentado en tres líneas de acción: la evaluación institucional (autoevaluación), la evaluación del sistema y los subsistemas de educación superior y la evaluación interinstitucional de programas académicos y funciones de las instituciones (De la Garza, 2007).

Para promover la evaluación externa, la CONPES creó en 1991 los Comités Interinstitucionales para la Evaluación de la Educación Superior (CIEES), como organismos de carácter no gubernamental. Las principales funciones asignadas a los CIEES fueron la evaluación diagnóstica de programas académicos y funciones institucionales y la acreditación de programas y unidades académicas. Los CIEES diseñaron metodologías y marcos de evaluación en cuya definición se tomaron en cuenta criterios y estándares internacionales. Hasta marzo del 2006 había evaluado casi 3000 programas académicos así como funciones institucionales. En 2006 identificaron 749 programas de licenciatura como acreditables (De la Garza, 2007).

En 2001 se creo el Consejo para la Acreditación de la Educación Superior (COPAES) y se inició la construcción de un Sistema para la acreditación de los programas educativos. La función del COPAES es regular los procesos de acreditación y certificar de la capacidad académica, técnica y operativa de las agencias de acreditación.

El COPAES ha otorgado reconocimiento a 16 agencias de acreditación de varias disciplinas y áreas del conocimiento como son: diseño, arquitectura, ciencias químicas, educación turística, ciencias sociales, enfermería, medicina, odontología, contaduría y administración, informática y computación, agronomía, medicina veterinaria y zootecnia, psicología, ingeniería, economía y ciencias del mar.

El número de programas acreditados ha variado de 156 en 46 instituciones en el 2002 a 774 en 108 IES en febrero del 2006. Desde el 2006 todo el territorio nacional cuenta con programas académicos evaluados por los CIEES y/o acreditados por las agencias reconocidas por COPAES. Además, las universidades privadas son certificadas por FIMPES y son susceptibles a la clasificación de "Institución de excelencia" por parte de la SEP, lo que les aseguran condiciones preferenciales de operación. 


\section{Retos del sistema de ES}

Los retos del sistema de ES son resultado en buena parte de su tamaño y complejidad. Algunos de estos retos son también consecuencia de la dificultad de lograr una coordinación efectiva de las políticas nacionales, estatales e institucionales, así como la discrepancia entre estas políticas y las costumbres, intereses, visiones y reglas del juego de los distintos actores.

Se podría enumerar un sinfín de desafíos del sistema de ES como la falta de recursos para financiar las instituciones públicas, la necesidad de ampliar y diversificar la oferta de una educación de calidad y pertinente, los problemas del personal docente, la creciente aparición de IES con animo de lucro que atienden a las expectativas de su mercado pero sin compromisos de formación como respuesta a las necesidades sociales.

En este sentido la transformación de la ES se tendría que orientar hacia la creación de un sistema abierto, flexible, innovador y dinámico, con una intensa colaboración interinstitucional y por la operación de redes para el trabajo académico que cubran amplios circuitos de los ámbitos estatal, regional, nacional e internacional. Además de fomentar la movilidad de profesores y alumnos entre instituciones, así como la búsqueda permanente de nuevas formas de enseñanza-aprendizaje.

En un ejercicio proactivo, a partir del análisis de la visión de la educación superior al año 2025 y en base a la situación actual del sistema, se pueden identificar los siguientes retos y estrategias para ES en México:

1. Para que las IES se conviertan en eficientes palancas impulsoras del desarrollo social, hace falta que:

a) Se aproximen académicamente al diagnóstico de las necesidades sociales existentes

b) En base a dicho diagnóstico, diseñen y operen programas de intervención comunitaria que:

- Incidan en los problemas más urgentes detectados.

- Aseguren la permanencia de la relación comunidad-IES

- Aseguren que la participación de los educandos en la operación de dichos programas de intervención redunde en el desarrollo de habilidades personales en su perfil profesional. 
c) Promover programas para el financiamiento que no estén ligados totalmente al resultado de los procesos de acreditación, sino también al papel que la IES está desempeñando en su entorno.

2. Para que las ES puedan proporcionar a los mexicanos elementos para su desarrollo integral, sería necesario:

a) Asegurar que los objetivos curriculares estén planteados en torno a la adquisición de competencias y habilidades, no solo de conocimientos. Esto es una tarea de las instancias certificadoras y de los grupos que participan en el diseño de los indicadores de calidad.

b) Establecer dentro de los planes de estudio el seguimiento y certificación del cumplimiento de actividades de aprendizaje de desarrollo de talentos.

c) Garantizar que las universidades destinen recursos, no solo físicos sino también humanos, para la consecución de dichos objetivos y operación de dichas actividades.

3. Para que las IES formen profesionales portadores de conocimientos de vanguardia, es imprescindible que las instancias certificadoras vigilen la eficiencia y auditen la calidad y la periodicidad de la actualización de planes y programas de estudio.

4. Para que las IES estén en posibilidad de atender una población que cubra a más de la mitad de la población entre 19 y 23 años, es importante:

a) Promover un crecimiento ordenado que asegure una calidad mínima en las nuevas instituciones, así como su rigor académico, sobre todo en las IES con ánimo de lucro que están proliferando en el país.

b) Idear instituciones de educación superior que puedan atender diversificada, flexible y pertinentemente a grupos sociales hasta la fecha excluidos de la posibilidad de obtener una educación de alto nivel y de buena calidad. 
c) Fomentar el uso eficiente de su capacidad instalada mediante una programación de sus actividades para responder mejor a la creciente demanda de ES.

d) Regular la oferta de programas educativos de manera que su diversificación permita realmente atender las necesidades del entorno, no solo respondiendo a las expectativas del mercado sino a las necesidades globales de la sociedad.

d) Normar y promover nuevos formatos educativos a través de medios no tradicionales, que permita la inclusión de personas geográficamente distantes o con poca disponibilidad de horarios.

5. Para lograr que las IES se encuentren en coordinación con otros tipos educativos, se hace necesario en general:

a) Auspiciar y fomentar la operación de asociaciones, consorcios y redes que permitan desarrollar sinergias.

b) Apoyar eficientemente en la profesionalización del personal docente de los demás niveles educativos.

c) Diseñar un programa con objetivos concretos que verifique el cumplimento del compromiso que las IES tienen como principales formadores de los profesionales académicos que operan y administran los demás niveles educativos.

Además en el ámbito de:

- La ciencia y la tecnología es preciso fomentar la vinculación empresa-IES para que la investigación y desarrollo que se realiza en las IES de verdad resuelva necesidades pertinentes al entorno productivo. Esta es, además una forma de inyectar recursos a las IES, ya que se les identificará como entidades eficientes de consultoría, de desarrollo de procesos y tecnología con capacidad real de resolución de problemas.

- En el fomento del arte y la cultura, es importante que más allá de las universidades interculturales, todas las IES participen en la preservación de la multiculturalidad del país, tanto en lo que se refiere 
a las lenguas indígenas como a las tradiciones y saberes ancestrales. Además de fomentar las actividades extra curriculares relacionadas con las artes.

6. Para que en las IES se promueva la movilidad de profesores y alumnos sería importante establecer, por perfil y nivel, cuotas mínimas de participantes en los programas y vigilar el cumplimiento de las mismas, en un esquema similar al PRONABES.

7. Para que las IES informen con transparencia a la sociedad respecto a su desempeño académico y el uso de sus recursos se podría:

a) Fortalecer el desempeño de la COPAES de tal forma que se consolide su tarea de difusión de los resultados de la acreditación de las IES.

b) Diseñar programas de capacitación para los administradores de las IES, que les permita profesionalizar su actividad diaria y ejercer con responsabilidad el liderazgo de las instituciones.

c) Mejorar los criterios de financiación, buscando una mayor equidad y transparencia en los mecanismos de otorgamiento de recursos a las IES, que al mismo tiempo garanticen la corresponsabilidad de las instituciones en el uso de estos recursos.

c) Aunque en el último sexenio se han diseñado estrategias nacionales para el fomento de la transparencia en el ejercicio público es imprescindible continuar en esta línea, sobre todo en las IES que obtienen fondos federales, para garantizar el uso correcto de estos recursos.

\section{Conclusiones}

La participación de las IES en los debates políticos y sociales del país ha sido una constante y tendría que mantenerse en el transcurso del tiempo. Más allá de la tradicional responsabilidad social, las universidades han contribuido al fortalecimiento de la democracia. Es importante que los gobiernos valoren esta contribución y apoyen el papel de las IES en el país a través de la toma de decisiones y el desarrollo de políticas que den solución a los grandes retos del sistema de ES. 
Hoy en día el país se enfrenta a importantes retos en el ámbito global, del conocimiento, social, económico y político. Todos estos desafíos requieren que las IES tengan una capacidad de respuesta y adaptación a la nueva realidad que a la vez se complica por la necesidad de recursos que les permita ejercer sus actividades de forma pertinente. La creatividad y la innovación de las IES en estos aspectos marcarán el rol que desempeñen en un futuro.

En un país de realidades tan heterogéneas como México en donde la mitad de la población vive en la pobreza, pero donde al mismo tiempo el $62 \%$ de las instituciones de ES son privadas, es un reto asegurar que dichas instituciones dirijan sus esfuerzos (definidos y gobernados intrínsecamente) hacia la formación de personas que tengan las capacidades, la motivación y el perfil necesarios para remediar las situaciones de indigencia de su entorno. La construcción de un México más justo y equilibrado socialmente necesita obligatoriamente la participación activa de las IES.

\section{Bibliografía}

ANUIES (2007), Consolidación y avance de la educación superior en México. Elementos de diagnóstico y propuestas. México D.F.

ANUIES (2005), Acciones de transformación de las universidades públicas mexicanas 1994-2003, Mexico D.F., (disponible en http://www.anuies.mx/ e_proyectos/pdf/LibAccionesdetransformacion1_05.pdf).

ANUIES (2000), La Educación Superior en el Siglo XXI, México D.F. (disponible en http://www.anuies.mx/servicios/d_estrategicos/documentos_estrategicos/21/index.html).

CINDA (2007). Educación Superior en Iberoamérica, Informe 2007. RIL Editores, Santiago de Chile, Chile.

Consejo para la Acreditación de la Educación Superior (COPAES) www. copaes.org.mx

De la Garza Javier en GUNI (2007). La educación superior en el mundo: Acreditación para la garantía de la calidad ¿Qué está en juego?. Mundi-Prensa. Madrid, España 
Didriksson, A., J. Fuentes Maya y A. Palma Cárdenas (2004), El Financiamiento para las Instituciones de Educación Superior en México 1990-2002. Trabajo presentado en el Seminario Regional sobre el financiamiento de la educación superior en América Latina y el Caribe, La Habana, Cuba, (disponible en www. iesalc.unesco.org.ve).

Grupo del Banco Mundial, América Latina y el Caribe (2004) Pobreza Extrema -Hoja informativa (disponible en http://wbln1018.worldbank.org/LAC/LAC. nsf/PrintView2ndLanguage/58371 FC96202347985256EDF005BBB99?Ope ndocument )

INEGI (2004) Serie boletín de estadísticas continuas, demográficas y sociales. Estadísticas de educación. Educación básica, media superior y educación Superior, p 25-34.

INEGI Estadísticas http://www.inegi.gob.mx/inegi/default.aspx

Schmelkes, S. (2005), Educación Superior Intercultural. El caso Mexicano. IESALC/UNESCO, Caracas, Venezuela.

Secretaría de Educación Pública (2005) Equidad, calidad e innovación en el desarrollo educativo nacional. SEP, capítulo 6, México.

Secretaría de Educación Pública, Subsecretaría de Educación Superior e Investigación Científica (SESIC) e Instituto Internacional para la Educación Superior en América Latina y el Caribe (IESALC) (2003), "Informe Nacional sobre la Educación Superior en México" (disponible en http://www.anuies. $\mathrm{mx} / \mathrm{e} \_$proyectos/pdf/01_Informe_Nacional_sobre_la_Educacion_Superior_en_ Mexico.pdf).

SEP Estadísticas ciclo escolar 2005-2006. http://www.sep.gob.mx

UNESCO (2006) Global Education Digest 2006. UNESCO-UIS 2006. ISBN: 92-9189-030-8. Canadá. 